\title{
Editorial. Facettes de l'(in)sécurité ou l'ordinaire de l'exceptionnel
}

Didier Bigo et Emmanuel-Pierre Guittet

\section{(2) OpenEdition \\ 1 Journals}

Édition électronique

URL : http://journals.openedition.org/conflits/954

DOI : 10.4000/conflits.954

ISSN : $1777-5345$

Éditeur :

CCLS - Centre d'études sur les conflits lilberté et sécurité, L'Harmattan

Édition imprimée

Date de publication : 1 septembre 2003

Pagination : 5-8

ISBN : 2-7475-5377-9

ISSN : 1157-996X

Référence électronique

Didier Bigo et Emmanuel-Pierre Guittet, « Editorial. Facettes de l'(in)sécurité ou l'ordinaire de l'exceptionnel », Cultures \& Conflits [En ligne], 51 | automne 2003, mis en ligne le 02 février 2004, consulté le 30 mars 2021. URL : http://journals.openedition.org/conflits/954 ; DOI : https://doi.org/ $10.4000 /$ conflits.954

Ce document a été généré automatiquement le 30 mars 2021.

Creative Commons License 


\title{
Editorial. Facettes de l'(in)sécurité ou l'ordinaire de l'exceptionnel
}

\author{
Didier Bigo et Emmanuel-Pierre Guittet
}

La revue Cultures \& Conflits s'est engagée dans une réflexion qui se traduit aussi par une présentation graphique légèrement différente apparue avec les deux précédents numéros sur le visa Schengen. Comme dans la mise à plat d'un ruban de Moebius, la maquette de présentation vise à rendre compte des processus et des dynamiques à l'œuvre dans notre monde contemporain fait de «frontiéralisations" et d'effets scalaires. Loin de «dualiser» et de penser en termes d'amis et ennemis, intérieur et extérieur, connu et inconnu, visible et invisible, la maquette graphique rend compte de notre orientation. Celle-ci est une tentative de présentation de ces stratifications étranges et multiples nécessitant une grille d'analyse alternative procédant d'une compréhension des variations spatiales et temporelles, des variations d'échelle, des jeux de mémoire et d'oubli, des effets de représentation et de mise en scène politique des événements.

Cette présentation du numéro de Cultures \& Conflits choisit aussi plus explicitement qu'avant de lier texte et choix des illustrations et de faire part des discussions de la rédaction. Ici, le choix d'un tableau réécrivant à sa manière la mémoire de la plus grande insécurité, celle de la vie déchue et de la mort organisée, vise à comprendre le lien entre l'état d'exception et la vie ordinaire. Donnez-moi la violence est une peinture grave aux couleurs criantes, dans laquelle se dégage le lointain souvenir morbide d'un camp de concentration, de son histoire et de ses histoires où violence, mépris, justifications voilées de sa réalité et survivances absurdes se mêlent et s'entremêlent dans notre mémoire. Certes le camp renvoie à priori à un univers concentrationnaire éloigné des pratiques des démocraties mais le camp, n'est-il qu'une image de la violence d'autres lieux et d'un autre temps? N'est-il pas aussi le dispositif par excellence de la suspension de l'ordinaire ? Celui qui « active » à la fois la mise au ban, les processus d'exclusion et leur routinisation, leur effacement, leur banalisation? N'est-il pas, plus encore que la prison ou le camp concentrationnaire, le symptôme contemporain matérialisé par des camps « ouverts» d'un impératif de circulation qui refuse les stocks et qui expulse, renvoie, fait circuler? Nous reviendrons dans un 
prochain numéro sur les dispositifs matériels des camps et de la mise à l'écart des étrangers mais ici nous voulons montrer qu'au-delà de la localisation du "camp ", les multiples dispositifs qui s'agencent de $l^{\prime}($ in)sécurité intérieure à l'(in)sécurité extérieure, de la violence dite criminelle à la violence dite politique des organisations clandestines, et des Etats avec la guerre, agissent à différents niveaux. Bien sûr, les effets scalaires jouent et la microphysique de la violence interindividuelle ne se lit pas de la même manière que les rapports de forces internationaux. Mais il existe néanmoins des «traces ", des lignes transversales, des "fragments » qui déstabilisent l'objectivation d'une frontière interne et externe et que l'on retrouve tant dans les mots que dans les choses, tant dans les relations que dans les processus. Cette frontière existe certes pour tout le monde mais elle est intersubjective et non plus objectivée une fois pour toutes. Elle ne ressort plus du cylindre traçant la différence interne/externe mais de la relativité du ruban de Moebius. Notre rapport à la violence est ainsi fait d'un ensemble d'images et de pratiques aux multiples facettes dont le prisme dépend du point de vue aléatoire, et parfois tournoyant, de l'observateur alors même que cela lui paraît si ordinaire, si naturel de signifier la différence entre les formes de violence, la distinction entre sécurité et insécurité. Seulement dire la sécurité, n'est-ce pas partir d'une image et d'une connaissance particulière et parfois arbitraire de la différence entre la violence et ce qu'elle ne serait pas ? De même, dire l'insécurité par opposition à la sécurité suppose de croire à une théorie des vases communicants où l'insécurité diminue quand la sécurité augmente. Mais est-ce le cas? La sphère virtuelle de l'insécurité n'est-elle pas l'enveloppe des processus de sécurisation et n'augmente-telle pas au fur et à mesure que l'on cherche à sécuriser? Quel rapport entretenonsnous à l'inconnu, à l'incertain, au futur? L'(in)sécurisation comme processus d'extrapolation du danger menaçant, de la peur de ce qui pourrait être mais qui n'est pas encore devient central dans nos sociétés contemporaines. Elle induit souvent une perte de perspective et la "réassurance » autour de mythes simplificateurs, construits autour de connaissances partielles, et d'anticipations institutionnelles ou collectives d'une violence exceptionnelle oubliant activement la violence ordinaire qui ne se lit plus comme telle.

Les articles de ce numéro de Cultures \& Conflits s'entrechoquent et néanmoins entrent en résonance. Ils se réfèrent, de différentes façons et selon les objets d'études qui les génèrent, à une théorisation de l'(in)sécurisation comme processus social et politique dont, parfois, ils donnent à l'avance une piste de recherche qui va au-delà de la frontière qu'ils avaient pensée pour leur objet mais qui n'offre aussi qu'un fragment, qu'une facette. En parcourant le spectre de la sécurité individuelle quotidienne des lieux défavorisés (L. Bonelli), des processus de mobilisation politique, des technologies de contrôle des mobilisations populaires, (A. Tsoukala, F. Jobard) des formes de surveillance des activités des organisations clandestines jusqu'aux mécanismes juridiques et politiques définissant la souveraineté et les moments de l'Etat d'exception, des rapports de la force et du droit, des relations de la justice à l'Etat et à la violence, des rapports de puissance et de leur dimension éthique (E. Guild, G. Devin), le lecteur aura sans doute l'impression, tout comme nous, d'une hétérogénéité dérangeante en ce qu'elle n'est pas sans articulation, sans point de rencontre. Aussi ce numéro construit d'articles divers a-t-il peut-être autant de cohérence que d'autres numéros de la revue en ce qu'il pointe l'ordinaire de l'exceptionnel, sur lequel nous reviendrons dans le prochain numéro. 
INDEX

Mots-clés : (in)sécurité 